
internationales

vol. $19-n^{\circ} 2$ | 2003

Les initiatives de l'étranger et les nouveaux cosmopolitismes

\title{
Entre Maghreb et Turquie : mobilités et recompositions territoriales
}

\section{Hélène Delos}

\section{(2) OpenEdition \\ Journals}

Édition électronique

URL : https://journals.openedition.org/remi/449

DOI : $10.4000 /$ remi.449

ISSN : $1777-5418$

Éditeur

Université de Poitiers

Édition imprimée

Date de publication : 1 juillet 2003

Pagination : 47-67

ISBN : 2-911627-34-2

ISSN : 0765-0752

Référence électronique

Hélène Delos, «Entre Maghreb et Turquie : mobilités et recompositions territoriales », Revue européenne des migrations internationales [En ligne], vol. 19 - n² | 2003, mis en ligne le 19 avril 2007, consulté le 14 avril 2022. URL : http://journals.openedition.org/remi/449 ; DOI : https://doi.org/ $10.4000 /$ remi.449

Ce document a été généré automatiquement le 14 avril 2022.

(c) Université de Poitiers 


\title{
Entre Maghreb et Turquie : mobilités et recompositions territoriales
}

\author{
Hélène Delos
}

1 L'évolution des migrations internationales et parallèlement des espaces urbains récepteurs de ces circulations ouvrent vers de nouvelles pistes d'observations et d'analyses dans le domaine des sciences sociales et humaines. Ces orientations de recherche illustrent un souci de mieux cerner une réalité migratoire et des formes de territorialisation plus complexes. Le cas des Maghrébins ${ }^{1}$ à Istanbul révèlent de nouveaux choix de destination et de localisation ainsi que des modes d'ancrage dans la ville moins déterminés comme dans le passé par les attaches historiques et économiques qui liaient le pays d'accueil au pays d'arrivée.

2 Notre article s'appuie sur des enquêtes de terrain réalisées à Istanbul, auprès de migrants originaires du Maghreb. Elles visent dans un premier temps à cibler cette population migrante, à comprendre son organisation spatiale ainsi que les étapes et les événements qui ont structuré cette chaîne migratoire. Elles permettent également une lecture plus fine des lieux en mouvement, des mouvements dans la ville et de la ville, au regard des pratiques sociales et spatiales des migrants. La métropole stambouliote est un pôle d'attraction principal pour ces migrants qui ont peu à peu marqué leur passage dans des lieux d'accueil et marchands.

3 Les circulations de migrants observées entre le Maghreb et la Turquie sont incomparables, en termes quantitatifs, avec les flux traditionnels orientés vers l'Europe. Elles ne s'inscrivent pas non plus dans une logique d'immigration mais dans un jeu permanent de passage entre deux ou plusieurs pays, exploitant ici et là les différentiels de richesse entre le pays d'origine et les pays d'accueil ou de passage. Notre recherche s'inscrit dès lors dans un paradigme mobilitaire où l'analyse de la mobilité dans ses composantes sociales et spatiales permet de mieux saisir la réalité des compositions ou recompositions urbaines en cours tant dans les pays de départ que dans les villes d'accueil ou de passage. Ces mobilités, aussi multiples soient-elles, 
s'imbriquent et se conjuguent dans le temps. La lecture de ces territoires de la mobilité, leur formation et les nouvelles conduites qu'ils génèrent, nécessite donc la prise en compte du facteur temps :

- temps des parcours individuels de migrants qui, poussés par le chômage dans leur pays d'origine et le manque de perspective, empruntent les routes de l'exil pour se rejoindre à Istanbul à la fin des années quatre-vingts : c'est le cas des résidents, Algériens et Tunisiens, aujourd'hui relais entre les grossistes turcs et les clients du Maghreb ;

- temps des rencontres et des «partenariats » entre ces résidents et une autre population migrante, turque et originaire du Sud-Est anatolien, qui a investi en moins de deux décennies dans le commerce international de textile au fur et à mesure des vagues successives de migrants dans la ville;

- temps de la mise en place des réseaux et d'un couloir migratoire à ce jour régulièrement emprunté entre les deux pôles géographiques que sont la Turquie et le Maghreb.

L'apparition de ce phénomène migratoire témoigne aussi d'un système-monde en mutation où l'expansion d'une économie mondiale redéfinit découpages et ouvertures de nouveaux territoires ; s'y imbriquent le contexte international - l'effondrement du bloc soviétique qui ouvre la Turquie vers de nouveaux horizons économiques, la difficulté d'obtenir un visa pour pénétrer l'Europe de Schengen - et les contextes nationaux, politiques ou économiques, justifiant les libertés d'action ou de contraintes auxquels les migrants font face. C'est dans ces interstices que les acteurs - " nomades » ou « sédentaires"- que nous avons ciblés vont chercher des ressources là où la mobilité, entendue comme stratégie, favorise une promotion sociale par la complémentarité d'espaces rendus de plus en plus proches grâce au développement des transports de masse.

Lecture d'une dynamique migratoire et de ses implications spatialesTerritoires et réseaux de la mobilité

5 L'approche pluridisciplinaire s'impose dans la mesure où l'analyse des recompositions territoriales et des flux migratoires qui les motivent traverse l'ensemble du champ social.

6 Nous avons réalisé nos premières enquêtes à Istanbul au cours de l'année 2001. Nos observations se sont d'abord centrées sur un quartier d'accueil d'une population migrante. Le quartier de Laleli voit affluer des Algériens, des Tunisiens et des Libyens dont la présence succède à l'arrivée des Iraniens depuis la révolution iranienne de 1979, des Arabes du Moyen-Orient dans les années 1980 (Irakiens, Syriens), des ressortissants des pays de l'Est puis des Russes dans les années 1990. Ils s'adonnent à un commerce informel entre Istanbul et leur pays d'origine. Cette activité commerciale autour d'un commerce dit «de la valise» (bavul ticareti) s'est développée dans les alentours du Grand Bazar mais aussi vers d'autres espaces de la ville en moins de deux décennies, à travers une circulation ininterrompue de migrants. Ils réactivent en un sens la tradition commerciale et artisanale du vieux Stamboul, héritée de la ville ottomane, en y déplaçant peu à peu le périmètre vers des espaces desservis par l'axe du tramway qui relie Beyazit à Zeytinburnu.

7 Comment ces populations ont-elle convergé en ces espaces communs pour y créer une centralité commerciale dont les effets se répercutent jusque sur les marchés locaux d'Alger et de Tripoli? C'est en termes d'opportunités, de formation de réseaux relationnels et de production de territoires de la mobilité vers des horizons toujours plus prometteurs que la réponse trouve une certaine pertinence pour cerner la réalité 
complexe de ce phénomène migratoire, si discret soit-il dans la métropole stambouliote.

8 Les groupes de migrants séjournant dans la ville, de quelques jours pour certains à plusieurs années pour d'autres, ont pour les plus âgés déjà acquis une expérience migratoire dans plusieurs autres villes du Moyen-Orient ou en Europe. Ils ont développé un savoir-faire dans la recherche d'opportunités de travail ou de commerces basé sur la mobilité, la connexion à un réseau de relations et la connaissance des lieux d'échanges. Souvent minoritaires et marginalisés par rapport à leurs activités informelles dans les pays de départ (comme les trabendistes régulièrement pointés du doigt dans la presse algérienne ou les "profiteurs du système » en Libye) comme dans celui d'arrivée, ils acquièrent un statut social plus valorisant dans un territoire qu'ils maîtrisent et à l'intérieur duquel savoir-circuler ${ }^{2}$ est une compétence partagée et reconnue. Le migrant «apparait donc comme plus libre de projet, sa référence est le territoire qu'il construit par ses parcours, ses haltes, ses traversées, sans se soucier outre mesure de sa naturalisation dans les valeurs ou usages qui outrepassent ses sociabilités quotidiennes » (Tarrius, 2000).

Le concept de "territoire circulatoire », développé par A. Tarrius, nous est apparu plus pertinent face à ces rapports à l'espace et au temps, puisqu'il prend en compte l'ensemble des réseaux, des relations sociales et des pratiques spatiales qui créent du territoire. Il nous aide à approfondir en géographie notre lecture de ces bouts de villes en mouvement sans réduire la notion de territoire à une portion d'espace approprié, continu, et strictement délimité. Ce concept donne un sens social au mouvement en restituant au migrant son rôle d'acteur et sa capacité à rebondir vers d'autres horizons en fonction de ses objectifs personnels et de l'apparition de nouvelles opportunités. Nous commencerons par un des pôles constitutifs de ce système migratoire, la métropole cosmopolite d'Istanbul.

Nouvelles centralités

À Istanbul, des touristes européens et des passants stambouliotes se rendent sur les lieux touristiques du vieux Stamboul, au sud de la corne d'Or, ignorant souvent qu'à deux pas du Grand Bazar, le centre se prolonge jusqu'aux quartiers de Laleli, de Beyazit et d'Aksaray, véritable complexe commercial pour un autre type de «touristes» (Carte 1). Souvent méconnu par la population autochtone, cet espace métropolitain figure comme une centralité pour ces «Russes »33 Maghrébins et, phénomène plus récent, pour ces Maliens, Sénégalais aperçus dans les ruelles commerçantes et attendus chaque jour par les commerçants de Laleli. Car c'est exclusivement vers une clientèle migrante que ces boutiques de confection et ces commerces de l'hôtellerie et de la restauration se sont orientés. 


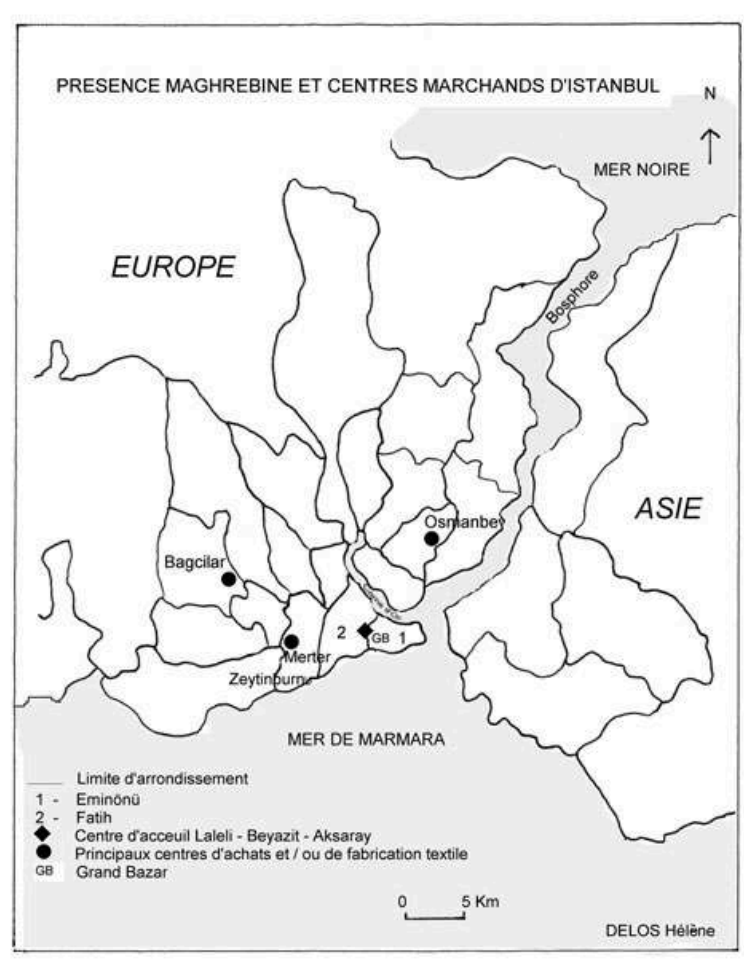

11 L'historique du quartier de Laleli permet de poser quelques repères au niveau des vagues successives de migrants qui l'ont peu à peu transformé. Ancien quartier résidentiel de classes moyennes, avec ses petits commerces de proximité, son tissu urbain s'est transformé avec la percée de grands axes automobiles (dont Ordu Caddesi) sous le gouvernement de A. Menderes, et l'arrivée de populations issues de l'exode rural. Dans les années quatre-vingts, c'est une population kurde arabophone et arabe $\mathrm{du}$ Sud-Est anatolien qui devient majoritaire dans le quartier. Les migrants sont originaires de Mardin, d'Urfa, de Diyerbakir ou encore de Batman. Des migrants kurdes rejoignent de la famille implantée à Aksaray ou à Beyazit. La population arabe originaire de ces régions du Sud-Est de la Turquie a afflué dans les années quatre-vingts après le coup d'État militaire puis dès 1991 après la fermeture des frontières avec l'Irak, à la suite de l'embargo ; beaucoup d'entre eux avaient acquis une expérience dans les activités de change ou dans le transport de part et d'autre des frontières irakiennes et syriennes (Deli, 2000). Istanbul est alors devenue une destination propice à de nouvelles opportunités.

À ce contexte s'ajoute un renouveau des rapports officiels, essentiellement économiques de la Turquie, avec les pays arabes dont certains pays du Maghreb depuis les années soixante-dix (Picard, 1993). Cette volonté, de la part de la Turquie, de développer ses échanges voire de renouer politiquement avec le monde arabe, s'est illustrée par son adhésion à l'Organisation de la Conférence Islamique en 1976 dont sont membres tous les pays arabes. Au début des années quatre-vingts, la libéralisation économique impulsée par Turgut Özal - «fruit d'un mélange culturel américain, dans le domaine économique, et islamique » (El-Zein, 1998: 138) - a relancé les échanges extérieurs avec le Maghreb ${ }^{4}$ et les pays pétroliers. Les entrepreneurs turcs ont alors fait leur apparition au Maghreb (notamment dans le secteur de la construction). Ils ont favorisé des rencontres entre Turcs (entrepreneurs essentiellement, surtout en Libye, drainant une main-d'œuvre issue des migrations inter-maghrébines) et Maghrébins. 
Alors qu'une main-d'œuvre turque émigrait vers les pays pétroliers dont la manne financière permettait le développement d'infrastructures, les ressortissants de ces nouveaux pays riches, dotés d'un pouvoir d'achat sans précédent, commençaient à affluer à Istanbul. C'est ainsi qu'à proximité du Grand Bazar, le quartier de Laleli vit apparaître ces riches touristes (Libyens, Irakiens et originaires du Golfe) et se reconvertit progressivement vers l'accueil de cette population dans le domaine de l'hôtellerie et la restauration (Keyder, 1998). L'investissement dans le commerce du textile international s'avère par la suite particulièrement lucratif, dans une période où la Turquie se lance dans une économie de marché. Les migrants des ex-pays communistes et les Russes viendront grossir ces premiers flux migratoires déclenchant le développement d'un véritable marché informel autour du textile. Les Algériens, Libyens et Tunisiens viendront par la suite s'ajouter plus discrètement à ces flux. Le textile est en effet l'une des principales branches industrielles de la Turquie et la confection l'une des spécificités commerciales d'Istanbul. La qualité et les prix bon marché ont vite attiré ces migrants, nouveaux commerçants en quête de produits de consommation rares dans leur pays, dynamisant les marchés, les sociétés d'importexport du textile, les usines de fabrication, sans compter les nombreuses boutiques de distribution.

13 Le commerce de la valise s'est fait plus visible et plus dense de part et d'autre de frontières désormais ouvertes à ces flux de «touristes » (Blascher, 1996). Le commerce informel désigne les migrants venus faire quelques achats à Istanbul pour les revendre sur des marchés locaux ou à des commerçants, une fois de retour dans leur pays d'origine. Estimé au milieu des années quatre-vingt-dix entre cinq et dix milliards de dollars ${ }^{5}$ (soit presque l'équivalent du tiers des exportations officielles turques évaluées à 27 milliards de dollars) il devient alors une caractéristique essentielle de l'économie stambouliote, agences de voyages, compagnies de bus et lignes aériennes se développent, accompagnant le mouvement. Des commerçants turcs apprennent des rudiments de langue russe, mettent à profit leur pratique de la langue arabe et embauchent des interprètes pour faciliter les transactions commerciales.

La constitution de ces centralités marchandes se situent donc au carrefour de ces migrations, et sont à l'origine d'une production territoriale inédite, toujours inachevée. Lieux de l'échange et échanges de compétences Istanbul au carrefour de nouvelles routes « touristiques »

15 Ville portuaire, prototype de la ville cosmopolite, Istanbul est un carrefour entre l'Europe, l'Asie et le monde arabe. Carrefour aussi de plusieurs civilisations, au regard d'un passé ouvert sur le brassage ou la cohabitation de plusieurs communautés. Tournés vers différents horizons en rapport avec ce passé ou actualisant de nouveaux destins en fonction d'intérêts touristiques et/ou économiques (Pérouse, 2000), les discours sur la métropole ont toujours fait référence à cette position particulière. Ils semblent occulter cependant une réalité migratoire qui imprime à la ville une urbanité éclairant davantage son avenir. Si la Turquie est traditionnellement connue comme une terre d'émigration, c'est surtout le poids de la métropole stambouliote qui change la donne, son attractivité en tant que ville de passage, de transit ou d'installation pour ces nouveaux venus d'horizons divers.

16 Ville plurielle, la mégalopole, aujourd'hui forte de plus de dix millions d'habitants, est à la croisée de migrations internes à la Turquie et internationales. L'exode rural massif amorcé dans les années 1960 a marqué la périphérie d'Istanbul par la prolifération des 
gecekondus. La rencontre de ces populations perçues comme "turbulentes " ${ }^{6}$ donne ici lieu à des cohabitations créatives, comme les interactions que nous avons observées entre une population maghrébine et des populations kurdes et arabes du Sud-Est de la Turquie.

Ces mobilités participent donc à l'internationalisation de la ville. "Ville-monde ", « relais international», «porte d'entrée et de sortie de la Turquie » (Pérouse, 2000), Istanbul ne concentre pas seulement des fonctions économiques et financières propres aux grandes métropoles de ce monde. J-F. Pérouse souligne aussi un autre trait du caractère international d'Istanbul, un "autre cosmopolitisme en gestation »: il s'agit de nouvelles populations résidentes, en transit ou de passage temporaire dans la ville (Iraniens, Bosniaques, Européens, Maghrébins, Arabes du Moyen-Orient, populations turcophones et autres populations d'origine géographique plus lointaine), dont les formes de cohabitation et d'insertion diffèrent quelque peu des figures d'un cosmopolitisme jusque-là associé à l'ancienne capitale d'empire.

Parmi toutes les formes de mobilité qui recomposent la ville, les mobilités transnationales révèlent une dimension particulière de cette mondialité. Moins stables que les migrations internationales de travail dans le cadre d'une période plus ou moins déterminée, elles s'appuient davantage sur des initiatives individuelles où le migrant choisit sa trajectoire spatiale et sociale. Ces initiatives reposent sur le profit que rapportent les passages fréquents de frontières, sur le balisage et le repérage des lieuxétapes qui ponctuent les couloirs migratoires; la frontière y assure une fonction régulatrice. Profit également recherché dans l'acquisition d'un statut social, à travers la maitrise d'un savoir-faire circulatoire comme condition d'ascension sociale. Ces logiques marchandes participent à la mondialisation des villes et à une économie où les lois du marché mondial sont utilisées ou contournées.

Dans la mesure où l'enracinement dans le pays d'accueil n'est pas l'objectif premier du migrant - celui-ci s'inscrivant dans des réseaux jouant sur plusieurs espaces et requérant des capacités à circuler entre ces espaces - c'est cette forme d'organisation socio-spatiale qui est novatrice dans ces rapports territoriaux. Les compromis qui se négocient entre une population locale et cette population migrante se rapprochent alors de la définition des "nouveaux cosmopolitismes » mentionnés par A. Tarrius (2000: 243-254).

Le « renouveau » d'une présence maghrébine à Istanbul ?

20 La présence maghrébine à Istanbul n'est pas nouvelle. Mais on peut parler de « renouveau » si l'on se réfère au XIX $X^{e}$ siècle où la présence de Tunisiens et d'Algériens avait été enregistrée à Constantinople dans les quartiers centraux de Beyazit ou à Tahtakale (Tunger-Zanetti, 1996 : 39-44 ; Bardin, 1979: 17). L'appartenance à ce passé commun lié à l'empire ottoman fait aujourd'hui référence pour un certain nombre d'acteurs, entrepreneurs turcs ou Maghrébins, que nous avons rencontré, dans la préférence et la confiance accordées à leurs clients ou partenaires commerciaux. La clientèle des pays de l'Est ne suscite qu'un intérêt purement commercial qui ne laisse place ni aux crédits ni à la confiance.

21 La fermeture de l'espace Schengen et les accords bilatéraux concernant la dispense ou l'obligation d'un visa pour les ressortissants de chaque pays du Maghreb, qui souhaitent entrer en Turquie, jouent également un rôle moteur dans l'impulsion donnée aux mouvements migratoires entre ces deux aires géographiques. 

mouvement d'abord discret et embryonnaire d'une population maghrébine vers Istanbul, rendue visible à mesure que se met en place une chaîne migratoire plus structurée entre le Maghreb et la Turquie. Autre mesure de cette visibilité: la transformation de certains espaces métropolitains tant dans les villes de départ qu'à Istanbul, dans lesquels les traces laissées par ces passages réguliers de migrants en remodèlent les contours et les formes, en redéfinissent les usages. Ces changements reflètent alors les marques d'une emprise et d'une appropriation davantage maîtrisées de ces espaces.

Premiers repérages dans un pôle du réseau migratoire et localiser la population migrante dans la ville. Ce choix présentait l'avantage de réunir tous les types d'acteurs (Turcs, Maghrébins, entrepreneurs, fourmis, résidents, commerçants) et de les différencier en fonction de la pluralité des situations migratoires observées. Notre objet que sont ces bouts de villes en mouvement se découvre alors au fil de nos rencontres avec les hommes, les femmes, au fil du repérage des espaces ou des lieux de leur vie quotidienne.

d'un premier séjour à Istanbul ${ }^{7}$, nous avons choisi une entrée par les lieux touristiques pour rencontrer une clientèle maghrébine ou des employés maghrébins. Devant l'emplacement de certaines boutiques ou échoppes touristiques, l'observation passive révélait un certain nombre de détails concernant les va-et-vient d'une clientèle à priori ciblée. Une première déambulation sur l'avenue de l'Indépendance (Istiklal Caddesi), rue piétonne du quartier de Beyoglu le long de laquelle s'alignent des magasins de luxe, nous a permis de voir quelques mots d'arabe ou de russe écrits discrètement dans le coin de deux ou trois vitrines de galeries commerciales. C'est toutefois de l'autre côté de la Corne d'Or, sur la rive Sud, que les premières investigations vont permettre d'identifier un réseau de lieux commerciaux dans la métropole. Car le migrant, commerçant ou fourmi ${ }^{8}$, qui débarque à Istanbul pour y effectuer des achats, dispose d'abord de quelques adresses dans le vieux Stamboul, centre historique et géographique de la ville, dans les quartiers de Laleli et Beyazit. Nous verrons comment cette première halte est justifiée par les qualités relationnelles qu'offrent ces quartiers; puis par quels liens ces espaces et les mobilités qu'ils génèrent sont à mettre en relation avec des espaces urbains du Maghreb.

Du point de vue de la méthode utilisée, notre objet nous a peu à peu dicté la marche à suivre pour remonter de l'observation directe, d'abord passive, à une analyse plus fine des réseaux et des relations qui organisent ces lieux de cohabitation. L'entrée dans les espaces du quotidien et de l'échange repose au préalable sur une certaine confiance justifiée notamment par les conditions d'existence de résidents au statut de "clandestins", donc susceptible de subir les frais d'une autorité policière. Les rencontres et les recueils de récits de vie nécessitent du temps, temps pour s'immerger dans les groupes puis pour être présenté à d'autres interlocuteurs. Cette démarche qui s'inspire de l'ethnographie s'appuie sur une observation participante où notre « implication » s'est parfois traduite en échanges de services.

Lieux et pratiques du quotidienLieux et indicateurs d'une présence maghrébine et d'une activité trans-territoriale

Les statistiques ne sont pas d'un grand secours lorsqu'il s'agit de dénombrer et de différencier la population maghrébine dans la ville. Simples indicateurs, ils fournissent 
des données générales et sont à manier avec précaution. Les chiffres obtenus par les renseignements de la Direction de la Sécurité d'Istanbul en 1999 révèlent l'existence de dix mille résidents officiels et dix mille résidents officieux (sans permis de séjour ni de travail) sans distinction d'origine, auxquels il faut ajouter cent mille entrées annuelles (Algériens, Libyens, Tunisiens) en référence aux visas de "tourisme». Le dernier recensement réalisé en octobre 2000 ne nous éclaire pas davantage : les portes des appartements sont restées closes pendant le couvre-feu pour les personnes sans permis de séjour. Nos rendez-vous auprès des consulats concernés nous ont permis tout au plus d'échanger informations et statistiques concernant les relations économiques et politiques entre la Turquie et les pays du Maghreb ; la population maghrébine présente à Istanbul ne concernant "que quelques familles et le personnel consulaire»; non mentionnée dans nos entretiens, l'activité de l'« autre présence maghrébine », moins officielle, est considérée comme occulte et peu honorifique. Miroir des réalités sociales des pays de départ, ces flux migratoires entre le Maghreb et la Turquie sont alors mis entre parenthèses. Des résidents de longue date nous ont expliqué qu'ils avaient fui le service militaire ou que leur enregistrement auprès des consulats ne leur était d'aucune utilité.

L'absence de permis de séjour ne signifie nullement absence de travail. Nous entrons là dans une économie dite " de l'ombre », favorisée au sommet de la chaîne migratoire par des entrepreneurs turcs ou maghrébins déclarés. Cette économie n'est donc pas ignorée par les différents gouvernements qui tolèrent ces activités pour les revenus qu'elles apportent à un certain nombre de migrants défavorisés ou touchés par le chômage dans leur pays d'origine.

Le Grand Bazar (Kapali Çarsi) à Eminönü, le Bazar égyptien (Mısır Çarsisi) et le quartier de Laleli entre les arrondissements de Fatih et d'Eminönü ont constitué le premier triangle d'observation. À la recherche de commerçants traitant exclusivement avec une clientèle du Maghreb, on nous indiqua un petit magasin d'or, tenu par un Turc arabophone originaire de Mardin. Les magasins d'or sont très nombreux dans une des grandes allées du bazar, mais nous avons fait mine de connaître et de chercher une boutique particulière en précisant que nous venions d'Algérie. Immédiatement, on nous conduisit dans la boutique de Sami. Celle-ci ne désemplit jamais dans la journée y compris durant les heures creuses, et l'étroitesse du magasin est accentuée par ces groupes de femmes accompagnées d'un parent, frère ou ami, qui se massent devant la porte. Sami est là depuis une quinzaine d'années, il a agrandi depuis peu sa boutique pour mieux canaliser et contrôler sa clientèle. Algériens, Tunisiens, quelques Libyens et Marocains forment l'ensemble de celle-ci. Dans sa boutique, nous rencontrons un couple d'Algériens qui après deux ans d'allers-retours entre Alger et Istanbul, achetant régulièrement de l'or, ont fini par investir, il y a huit mois dans un restaurant à Laleli. Ce projet n'a cependant pu se réaliser que grâce à un intermédiaire et associé turc.

Au Bazar égyptien, connu des tours opérateurs et partie intégrante de tous les circuits touristiques guidés, ce sont des employés que nous avons rencontrés. Plus de cinq employés travaillent dans les magasins d'épices et de confiseries, deux Marocains de Casablanca et de ses environs font partie des plus anciens avec cinq et dix ans d'expérience dans le domaine; embauchés pour leur aptitude à parler plusieurs langues, ils sont payés dix dollars la journée et pour certains logés dans un studio loué par le propriétaire de la boutique. Beaucoup ont leur bac, sont diplômés et attendent d'accumuler un revenu pour envisager de partir. Le séjour de ces migrants à Istanbul, 
au départ conçu comme temporaire, ne représente pour eux qu'une étape dans un parcours qu'ils souhaiteraient plus enrichissant. Ils savent que la concurrence est rude et doivent accepter les conditions de travail imposées par les employeurs.

Lors d'un deuxième séjour, cette fois accompagnés de deux interlocuteurs algériens que nous avons connus auparavant, nous avons visité d'autres boutiques spécialisées dans la confection et les tissus, en remontant le long des ruelles commerciales qui mènent $\mathrm{du}$ bazar égyptien au Grand bazar; là nous avons fait connaissance avec des femmes marocaines venues à Istanbul pour y trouver un travail. Nous y avons aussi rencontré un propriétaire turc qui après avoir travaillé une dizaine d'années chez Renault a choisi de s'établir à son compte dans la confection, mettant à profit sa parfaite maîtrise de l'arabe pour capter une clientèle maghrébine.

31 De l'autre côté de l'avenue Ordu caddesi qui donne sur Laleli, les devantures et le flot d'hommes et de femmes affairées, chargés de sacs rayés bien connus des commerçants à la valise indiquent un changement de rythme et de décor. Cette foule déborde souvent du quartier pour venir prospecter sur les petits marchés de détails qu'expose la grande place dominée par l'Université de Beyazit. Les enseignes portant la marque des chaînes de vêtements turques (Tom Martin, Cabani), particulièrement prisées en Algérie, Tunisie et Libye, rivalisent avec la boutique de Pierre Cardin; un bureau de change à des taux attractifs voit affluer les clients du Maghreb et des pays de l'Est. Les quelques enseignes en arabe, recouvertes par celles qui étaient en russe, refont leur apparition : elles désignent la boutique d'un grossiste, un restaurant aux préparations culinaires connues des clients (comme "La Casbah»), ou interpellent les plus gros chalands du haut d'une façade d'immeuble pour offrir les services d'un transitaire (Golfe, Aksan, Aslanlar). Parmi les premiers transitaires maghrébins d'Istanbul, certaines figures font référence comme celle d'un Algérien venu dans les années quatre-vingts faire fortune dans le textile et qui par la suite a fait commerce de pièces détachées. Accolé au quartier de Laleli, Aksaray complète le dispositif commercial et offrira éventuellement quelques divertissements nocturnes, pour ces migrants de passage, en affaires.

Des pratiques urbaines et des parcours migratoires différenciés

La connaissance et la pratique de certains espaces métropolitains stambouliotes par les résidents manifestent leur rôle d'intermédiaires entre fourmis, commerçants et grossistes. Ce positionnement social s'est construit après une période d'exil et d'errance plus ou moins longue pour ces anciens circulants. La prise en compte de leur parcours migratoire, de l'expérience acquise durant cette période jusqu'à ce qu'Istanbul figure comme une nouvelle balise urbaine, a permis de décomposer leur trajectoire jusqu'à leur rencontre avec les migrants turcs de Laleli.

L'acquisition du parc de logements par les Arabes et les Kurdes de Turquie, loués à des migrants d'Europe de l'Est et de Russie et aux Maghrébins, confirme leur domination des lieux et leur rôle dans l'accueil et la canalisation des flux de migrants. Ils se sont associés très tôt avec les premiers circulants (associés maghrébins pour la recherche et la gestion des stocks, et la captation de la clientèle). Hocine est Algérien, il est arrivé en 1994 à Istanbul, et habite Laleli. Comme d'autres pionniers, il est un témoin de la reconversion du quartier autour du commerce textile :

«Je suis arrivé en Septembre 1994. À Beyazit, il n'y avait pas encore beaucoup d'Algériens; j'étais le quatrième, je crois. J'ai d'abord habité à Taksim, puis à Eminönü, à Aksaray et enfin à Laleli. Au début quand je suis arrivé, les enseignes en arabe n'étaient pas aussi nombreuses. Il y avait juste le café algérien où j'ai 
travaillé. J'ai fait du commerce avec des vases afghans que je revendais à ceux qui passaient. [...] Après tout le monde s'est mis au textile. Tu vois ici dans cet immeuble, il n'y avait que des familles turques. Elles ont commencé à partir dès l'arrivée des Kurdes qui ont alors acheté ces appartements. Maintenant ils nous les louent à nous, aux Russes... [...] et plus personne ne s'occupe de l'entretien des immeubles... ».

34 À la clientèle russe, moldavienne, ukrainienne, roumaine s'est ajouté au début des années quatre-vingt-dix la clientèle maghrébine. Les Libyens apparaissaient déjà en petit nombre à la fin des années quatre-vingts alors que se développait en Libye le commerce informel, mais Istanbul était peu fréquentée, ceux-ci optant pour la proximité avec la Tunisie et l'île de Malte. À Tripoli, les vêtements de mode en provenance de Turquie sont également très prisés par une jeunesse libyenne dont les aspirations correspondent à un besoin d'ouverture vers l'extérieur. Moins présents à Istanbul par rapport à la population tunisienne, marocaine et algérienne durant ces dernières années, les « touristes » libyens se font plus nombreux aujourd'hui, aux dires d'un certain nombre de commerçants de Laleli, de Merter et d'Osmanbey qui traitent quasi exclusivement avec cette clientèle. La lecture du nombre de visiteurs maghrébins (les plus nombreux par rapport aux autres ressortissants arabes) en Turquie entre 2000 et 2001 le confirme :

Tableau $1:$ Interest of Arab turist in Turkey (\%) Istanbul

\begin{tabular}{|l|l|l|l|}
\hline Country & 2000 & 2001 & Increase (\%) \\
\hline \hline Tunisia & 68,817 & 72,143 & 4,83 \\
\hline \hline Algeria & 57,094 & 62,866 & 10,11 \\
\hline \hline Libya & 43,690 & 55,955 & 28,07 \\
\hline \hline Egypt & 27,048 & 30,364 & 12,26 \\
\hline \hline Jordan & 20,753 & 24,063 & 15,95 \\
\hline \hline Saudi Arabia & 19,102 & 19,102 & 35,93 \\
\hline \hline Syria & 13,057 & 13,963 & 6,94 \\
\hline \hline Lebanon & 12,092 & 17,567 & 45,28 \\
\hline \hline UAE & 3,089 & 3,535 & 14,44 \\
\hline \hline Yemen & 271 & 1,624 & 499,26 \\
\hline \hline Bahrain & 245 & 1,985 & 710,20 \\
\hline \hline Qatar & 43 & 548 & 1679,07 \\
\hline & 120 & \\
\hline \hline
\end{tabular}




\begin{tabular}{|l|l|l|l|}
\hline Kuwait & 643 & 7,971 & 1139,66 \\
\hline
\end{tabular}

Source : Anadolu Ajansi (22/02/2002) réouverture du guichet de Libyan Airlines qui propose aujourd'hui 3 vols par semaine (deux lignes Tripoli-Istanbul et une ligne Benghazi-Istanbul). À Laleli, c'est dans une galerie commerciale qu'ils effectuent leurs achats. Les propriétaires des locaux sont tous originaires de Mardin et les paquets entreposés chaque jour au rez-de-chaussée mentionnent tous en arabe leur destination vers la Libye. Lors des transactions commerciales, ce sont des Algériens et des Tunisiens, associés à des Turcs, qui se chargeront de négocier les prix et les commandes et de tenir la caisse. Car pour ces migrants de passage dans la ville, l'arabe est la seule langue de communication possible et l'on comprend alors en quoi la présence de Maghrébins sédentaires est souvent indispensable.

La présence de Tunisiens et de Libyens n'est pas nouvelle mais mieux canalisée et le rôle des Algériens est à souligner dans celui d'intermédiaires entre les grossistes, les fabricants turcs et les fourmis. Aujourd'hui sur-représentés dans la population maghrébine résidente, leur ancrage dans le quartier est plus important que celui des ressortissants des ex-pays communistes. Les relations qu'ils ont tissées avec les Turcs sont plus souples que les rapports entre Turcs et «Russes » décrits comme purement commerciaux et intéressés. Des entretiens avec des femmes ukrainiennes, moldaviennes et russes employées comme interprètes nous ont confirmé cette absence de fraternité voire un certain mépris des patrons turcs vis-à-vis d'elles ${ }^{9}$. Les responsabilités déléguées à des associés maghrébins au sein des boutiques de grossistes (gestion de la caisse, des stocks, des clients) les situent donc comme des partenaires privilégiés. Ces propos sont toutefois à nuancer dès que l'on sort du strict cadre commercial, comme le raconte A., associé dans une boutique :

«Les Turcs nous apprécient en tant que bons consommateurs. Les gens viennent pour dépenser et s'en vont. Par contre ceux qui restent leur donnent l'impression d'une invasion. Les Turcs manquent d'humanité, ils ne pensent qu'à l'argent... c'est "para, para, para"10 toute la journée... ».

Ce sentiment général s'explique aussi par leur relative liberté de mouvement, restreinte hors des zones commerciales. N'ayant pas renouvelé leur visa de tourisme (dont les mois de retard correspondent à un montant de plus en plus élevé qu'il faudra payer à la sortie du territoire), ils peuvent faire l'objet d'une arrestation et sont passibles d'une amende. La reconnaissance est donc toute relative en fonction des intérêts de chacun.

Une chaîne migratoire entre le Maghreb et Istanbul aurait-elle pu s'organiser sans la présence et l'accueil de ces pionniers ? C'est sur ce point que les résidents forment une catégorie particulière de migrants dont la sédentarité de plus ou moins longue durée leur a permis de tisser leur propre réseau de relations personnelles, de se familiariser au quotidien avec la langue turque, et surtout de repérer en quels lieux de la ville des opportunités pouvaient être saisies. Tous dotés de téléphones portables, ils sont souvent appelés par des fabricants ou des grossistes dès qu'un arrivage est susceptible d'intéresser leurs clients.

Apprentissage de la ville et acquisition d'un savoir-faire autonome 
39 Les démarches quotidiennes des résidents maghrébins les plus mobiles et les plus indépendants dans la ville nous ont introduits dans tous les espaces où se déroulent les échanges. Installés à leur compte, ils sont en contact avec des fabricants, des grossistes, et se déplacent pour commander, chercher de nouveaux modèles à proposer aux clients et acheminer les commandes jusqu'à leur appartement, converti en petites centrales d'achats. L'échelle quotidienne est alors riche de sens pour relever, au jour le jour, les déplacements d'un lieu à un autre, les échanges qui se dissimulent dans les appartements, les transactions commerciales et la manière dont s'effectuent ces échanges.

La reconversion du quartier de Laleli se prolonge jusque dans les espaces privés. H., comme ses voisins de l'immeuble, a réaménagé son appartement pour recevoir sa clientèle, entreposer les cartons, et exposer ses marchandises. Il le loue à quatre, cent dollars par mois et sous-loue trois chambres sur quatre à cent dollars à des Russes, des Ukrainiennes ou Moldaviennes. La proximité des logements avec l'ensemble des commerces rend les loyers aussi chers qu'à Taksim. La salle de séjour est destinée exclusivement à la réception de sa clientèle ; ici, on expose, on négocie, on commande, on stocke, on sert cafés et gâteaux puis on fixe une date de retour au client qui reviendra alors pour trois ou quatre jours chercher sa commande. Puis H. retournera prospecter auprès d'un fabricant ou d'un grossiste avec qui il est associé de manière informelle. Entre eux, les transactions se feront en turc. Nous avons suivi Hocine à Merter, à proximité de la zone industrielle de Zeytinburnu ; Merter est une vaste zone de production, de confection et de distribution via des galeries «Import-Export » ou des petites boutiques, où le haut-de-gamme comme les occasions satisfont tous les types de clientèle. C'est un centre textile fondé au milieu des années soixante-dix et qui s'est développé dès les années quatre-vingts avec l'ouverture de la Turquie au marché mondial. Il alimente également les grossistes de Laleli. D'une superficie de plus de quatre mille mètres carrés, ce centre de confections plus que de production fait travailler cinq cent mille personnes et trouve l'essentiel de ses débouchés auprès des pays européens, de l'Asie centrale, de l'Amérique et des pays arabes.

41 Il existe d'autres petits métiers qui se greffent sur ces activités et leur sont complémentaires: l'emballage des cabas, que l'on peut apercevoir dans les halls d'hôtels ou leur sous-sol, leur acheminement à l'aéroport, la répartition équilibrée des poids, pour ne pas dépasser les trente kilos autorisés selon les compagnies. Il existe aussi les petites sociétés de transports et tous les petits porteurs qui arpentent les ruelles du matin au soir, encombrant la circulation automobile aux heures de pointe. Les patrons des hôtels ont investi dans un mode de transport qui leur permettra ainsi de toucher une commission à chaque transport de bagages. À l'aéroport, les résidents qui assureront le reste du service toucheront une commission.

42 C'est dans les hôtels, les cafés et les appartements, seuls lieux de sociabilité pour les Maghrébins, que s'échangent les informations sur le pays d'origine, la mode du moment et l'évolution du contrôle dans les douanes. C'est ici qu'ensemble seront partagés les repas de fêtes, comme pour le ramadan, l'Aïd. Au cours de ces repas, nous avons rencontré des femmes et des hommes qui avaient choisi l'exil pour tenter leur chance ici. Mais aussi des touristes venus visiter le pays et rendre visite à des proches avant de reprendre le voyage organisé. Des jeunes mariés ont choisi la ville mythique comme décor de leur voyage de noces, mais c'est dans un hôtel chic de Laleli que se portera leur choix, pour ne pas manquer de saisir des occasions d'achats. Des femmes 
aussi, mariées avec des Kurdes puis divorcées, souvent mères de famille et contraintes de venir s'installer à Laleli pour trouver un travail dans les boutiques, dans une société d'import-export ou dans un restaurant. Elles trouveront dans le Laleli résidentiel, à proximité de la corniche, des loyers beaucoup moins chers qu'au cœur du quartier où se concentre l'ensemble des commerces. Ces quartiers kurdes abritent aussi des migrants moins fortunés (Iraniens, Marocains, Africains), parfois candidats au passage clandestin vers l'Europe.

D'un pôle urbain à un autre : villes en miroir

Lorsque au début des années quatre-vingt-dix, des Algériens et des Tunisiens décident de s'installer à Istanbul, la plupart d'entre eux ont déjà connu une expérience migratoire en Syrie et en Libye, voire en Europe. C'est ainsi qu'A., arrivé en 1989 à Istanbul s'est déjà rendu en Russie en tant que marin dans la marine marchande, et s'est familiarisé avec la langue russe ; il est allé en Syrie et a également passé quelques années en Libye, à Tripoli, durant la période où le pays signait des contrats avec des firmes turques de construction afin de se doter d'infrastructures. La main-d'œuvre était algérienne, tunisienne et c'est là qu'Ali a appris le turc. C'est aussi le cas de H., qui a quitté sa ville natale de Togourt en Algérie pour se rendre en Libye jusqu'à ce qu'il soit informé des opportunités de travail que pouvait offrir Istanbul :

«Je suis parti en Libye où j'ai habité à Tripoli puis à Ghadamès dans le sud. J'ai travaillé pour la municipalité dans le bâtiment puis dans un restaurant de Ghadamès [...], dans la contrebande aussi [...] je suis allé au Niger, au Mali où je vendais des pièces détachées de voitures venues d'Europe [...]. Quand j'étais à l'école, pendant quinze ans à chaque vacances je faisais du commerce [...]. J'ai aussi travaillé dans le textile, j'achetais à Benghazi, à Tripoli aux souks puis je revendais à Ghadamès. Il y en a qui font des études, moi j'ai appris en regardant autour de moi, partout, j'ai regardé les goûts, la mode à la télévision et c'est comme ça que je me suis vite adapté au commerce à Istanbul [...]. J'ai entendu parler d'Istanbul quand j'étais en Libye par des Libyens qui partaient régulièrement à Istanbul. Après, comme beaucoup d'étrangers, j'ai été expulsé de Libye et c'est comme ça que je suis venu à Istanbul en 1994... Aller en Europe ? J'y pense, mais je préfère vivre confortablement ici dans le commerce qu'être pauvre en Europe ».

44 Le parcours de K., arrivé en 1998 à Istanbul, rejoint celui d'un certain nombre de résidents qui ont transité en Syrie. Il a quitté l'Algérie en 1990 puis est parti en Arabie Saoudite pour poursuivre ses études. Comme son permis n'autorisait qu'un séjour d'études, il a quitté l'université après son diplôme de langue arabe pour aller en Syrie :

«En Syrie, j'ai fait un peu de commerce et je donnais des cours d'arabe classique... Après je n'ai pas pu résister... Tout le monde ne parlait que de ça alors j'ai pris le bus et je suis venu à Istanbul [...]. J'ai habité d'abord à Taksim, chez un ami allemand que je connaissais de Damas. Puis le lendemain matin, nous sommes sortis pour voir la ville. Après, on est passé au Bazar égyptien, j'ai rencontré un Tunisien là-bas [...], il m'a dit qu'il y avait un endroit où je pouvais rencontrer des Algériens, à Beyazit. Comme je ne connaissais pas, il m'a emmené là-bas et j'y ai rencontré un Algérien que j'avais connu en Syrie et qui faisait du commerce entre Istanbul et Damas, il achetait à Istanbul et revendait à Damas [...]. On a pris un thé ensemble et je lui ai posé des questions sur la vie ici. Il m'a dit de me reposer trois jours puis de revenir le voir. Pour ça, c'est bien entre Algériens, il y a une grande solidarité... Un autre jour, je suis revenu seul et j'ai cherché le café algérien dont j'avais entendu parler en Syrie. J'ai retrouvé un ami de ma ville natale. Du coup, il m'a dit «si tu veux travailler, pas de problèmes, avec le temps tu apprendras à faire du commerce » [...]. Pour me loger? Je revenais de Beyazit et je suis allé dans une épicerie à Hortakoy. J'ai vu un Marocain, je ne le connais pas bien, mais on s'est vu en Syrie. Je lui ai demandé s'il savait où je pouvais trouver une chambre à louer. Il 
m'a donné la carte du propriétaire de l'appartement où il vivait. Il est Turc, il parle arabe [...]. » misatoire correspondait à la dispense de visas pour tous les ressortissants arabes ou d'origine arabe. Pour les Algériens, c'est une des destinations qui s'est imposée après la montée du chômage dans les années quatre-vingts en Algérie, la Syrie offrait les moyens de poursuivre des études pour un certain nombre d'étudiants. Elle offrait aussi un refuge pour des sympathisants islamistes. A. y a poursuivi ses études, il habite aujourd'hui dans un hôtel de Laleli à Istanbul :

«Je suis arrivé en 1994 à Damas en transitant par Tripoli en Libye, à cause du chômage en Algérie. À Damas, j'ai poursuivi mes études de langue à l'université. J'ai tout abandonné après deux ans d'études car il me fallait bien un métier à côté pour vivre. J'ai commencé à donner des cours mais les problèmes ont commencé à arriver dès 1995, avec la visite de Zéroual en Syrie, pour traquer les «islamistes " [...]. Il y a eu des abus et l'on pouvait être dénoncé pour des histoires de jalousies dès qu'on était Algérien. Si on arrivait juste à Damas, on recevait une convocation et on devait se présenter le lendemain [...]. Là, ils disaient aux Algériens de rester peu de temps. Alors comme les lois ne sont pas claires ni bien définies, il vaut mieux partir sinon on peut disparaître subitement. J'avais entendu parler d'Istanbul alors je suis venu ici et maintenant je fais un peu de commerce et j'achemine les marchandises à l'aéroport pour équilibrer les bagages de mes clients ".

À partir de 1996, les affaires deviennent florissantes à Istanbul. Les commerçants du Maghreb affluent et le montant des commandes peut atteindre plus de trois cent mille francs. Aujourd'hui, l'activité commerciale est quelque peu ralentie (Péraldi, 1998) accentuée par une baisse de la clientèle russe ${ }^{12}$. Il y a deux ans, $\mathrm{K}$. pouvait travailler tous les jours pendant deux mois, ce qui n'est plus le cas aujourd'hui. Pourtant malgré la crise économique et l'inflation qui frappent la Turquie depuis février 2001, un magazine turc compare les revenus du textile durant les saisons pleines à un an 
d'intervalle et annonce des bénéfices croissants, soulignant l'intérêt à conserver et mieux accueillir la clientèle ${ }^{13}$.

Les premiers circulants ou pionniers, - entrepreneurs turcs et maghrébins partis faire fortune à Istanbul dans les années quatre-vingts et nomades maghrébins en quête de sédentarité passagère -, ont ainsi contribué à baliser des routes migratoires et favoriser l'accueil des fourmis en se maintenant en position d'intermédiaires entre les clients de passage et les grossistes ou fabricants d'Istanbul. Tous ne sont pas restés longtemps dans la métropole turque comme les plus diplômés, médecins, ingénieurs, journalistes, surtout Algériens, venus à Istanbul depuis la montée du chômage et les premiers événements. Ceux-là ont trouvé refuge au Canada, en Angleterre, après la décision pour certains d'entrer clandestinement en Europe, via la Grèce. D'autres s'interrogent sur la possibilité de reprendre la route, si leur place au sein de ce vaste dispositif venait à être remise en question. Certains plus âgés souhaiteraient s'installer à Istanbul pour y monter un projet, mais sont freinés par les procédures administratives qui les placent en situation de dépendance et leur imposent de partager une grosse partie des gains financiers avec leurs associés turcs.

Conclusion

Les flux d'hommes et de femmes qui circulent aujourd'hui entre le Maghreb et la Turquie font de l'urbain l'horizon privilégié des recompositions territoriales qui accompagnent ces mouvements. Sur l'initiative de commerçants, d'entrepreneurs et d'une jeunesse ayant connue l'exil ou l'errance, l'appropriation de certains espaces stambouliotes bâtis sur la maîtrise d'un tissu relationnel de plus en plus dense a permis que s'ébauchent puis s'affirment des territoires réticulaires. Cette mobilisation des migrants algériens et libyens dynamise les marchés et les petits commerces d'Alger et de Tripoli désormais remplis de produits turcs - pour ne citer que les capitales comblant les besoins d'une population avide de produits de consommation.

51 Mais la présence des migrants maghrébins à Istanbul comme le rôle des résidents n'estil pas voué à disparaître? Ce que l'on peut dire c'est que le commerce de la valise à Istanbul reste dominant dans l'activité des migrants. Et si la capitale économique et culturelle turque figure de plus en plus dans les prospectus touristiques au Maghreb, la venue de petits commerçants migrants peut, elle, être remise en question dans les années à venir. L'évolution du contexte international, la concurrence de nouvelles places commerciales et la recherche d'opportunités toujours plus attrayantes sur le plan économique comme en termes de cadre de vie risquent de pousser ces migrants vers de nouvelles balises urbaines.

52 Ces espaces du mouvement qui «situent le monde dans le lieu » et redéfinissent des formes de centralités « en les tirant du lieu vers le monde » (Tarrius, 2000 : 132) n'en révèlent pas moins à notre sens un rapport au temps et à l'espace propre à notre époque. 


\section{BIBLIOGRAPHIE}

BARDIN Pierre (1979) Algériens et Tunisiens dans l'Empire ottoman de 1848 à 1914, Paris, CNRS Éditions.

BAZIN M., KANÇAL S., PEREZ R., THOBIE J. (1998) La Turquie entre trois mondes, Varia Turcica XXXII, Paris, L'Harmattan, 450 p.

BELANGER Pierre et alii (2000) Faire Dubaï, in Francine Fort (dir.), Mutations, Barcelone, Éd. ACTAR, pp. 710-714.

BLASCHER P. (1996) Les shop-turisty de Tasargard ou les nouveaux russophones d'Istanbul, Turcica, T.28, pp. 11-50.

BOUHAMIDI Mohamed, Trabendistes, in Thierry Fabre (dir.), La pensée de Midi, $\mathrm{n}^{\circ} 4$, Printemps 2001, Éd. Actes Sud/La pensée de Midi, pp. 12-16.

DELI Fadime (2000) Les flux migratoires des populations originaires de Mardin vers Istanbul, in Isabelle Rigoni (dir.), Turquie : les mille visages (politiques, religion, femmes, immigration), Éd. Syllepse, pp. 117-130.

DE TINGUY Anne (2000) Le phénomène des «Tchelnoki » et la constitution d'espaces transfrontaliers dans la région de la Mer Noire, in Marcel Bazin et alii, Méditerranée et Mer Noire entre mondialisation et régionalisation, Actes du colloque international d'Antalya, 11-12-13 Septembre 1997, Paris, l'Harmattan.

HILY Marie-Antoinette, MA MUNG Emmanuel et alii. (1998) La circulation migratoire in Migrations Etudes, $\mathrm{n}^{\circ} 84,12 \mathrm{p}$.

GANGLOFF Sylvie, PEROUSE Jean-François (2001) La présence roumaine à Istanbul. Une chronique de l'éphémère et de l'invisible, Observatoire Urbain d'Istanbul, les dossiers de l'I.F.E.A., $\mathrm{n}^{\circ}$ 8, Octobre 2001,47 p.

KEYDER Ç (1997) Laleli's quick-change acts, in K. Brown, R. Waterhouse Éds., Istanbul, un monde pluriel, Méditerranéennes $n^{\circ}$ 10, pp. 175-181.

MARTINEZ Luis (2000) Libye : transformations socio-éco et mutations politiques sous l'embargo, Paris, Éditions des Sciences politiques, $28 \mathrm{p}$.

MAZZELLA Sylvie (1996) La ville-mémoire. Quelques usages de la Mémoire collective de Maurice Halbwachs, Enquête, 4, pp. 177-189.

PERALDI Michel (1998) Le cycle algérien dans Laleli morose, Observatoire Urbain d'Istanbul, Lettre d'information $n^{\circ} 14$, I.F.E.A, pp. 19-22.

PEROUSE Jean-François (2000) Istanbul : une métropole méditerranéenne ? Critique d'un lieu commun tenace, Colloque de Grasse, $21 \mathrm{p}$.

PEROUSE Jean-François (2000) L'internationalisation de la métropole stambouliote : expressions et limites, in Marcel Bazin et alii, Méditerranée et Mer Noire entre mondialisation et régionalisation, Actes du colloque international d'Antalya, 11-12-13 Septembre 1997, Paris, L'Harmattan.

PICARD Elisabeth (dir.) (1993) La nouvelle dynamique au Moyen-Orient. Les relations entre l'Orient arabe et la Turquie, Paris, L'Harmattan, 215 p.

SIMON Gildas (1995) Géodynamique des migrations internationales dans le monde, Paris, P.U.F, 430 p. 
TARRIUS Alain (1989) Anthropologie du mouvement, paris, Éd. Paradigme, 185 p.

TARRIUS Alain (1992) Les fourmis d'Europe. Migrants riches, migrants pauvres et nouvelles villes internationales, Paris, L'Harmattan, 207 p.

TARRIUS Alain (2000) Les nouveaux cosmopolitismes. Mobilités, identités, territoires, Éd. de l'Aube, $266 \mathrm{p}$.

TUNGER-ZANETTI A. (1996) La communication entre Tunis et Constantinople : 1860-1913. Province et métropole, Paris, L'Harmattan, $303 \mathrm{p}$.

\section{NOTES}

1. Nous traiterons ici plus exclusivement des Algériens et des Libyens.

2. Selon la terminologie employée par A. Tarrius.

3. Ce terme est fréquemment utilisé pour désigner les personnes originaires des pays de l'Est et de l'ex-URSS.

4. Le volume des échanges commerciaux montre que l'Algérie est le principal partenaire commercial de la Turquie (et le premier partenaire africain), suivi par la Libye et la Tunisie, puis par le Maroc. Ülkelere göre dis ticaretimiz, 1997, 1999, 2000. Statistiques du gouvernement.

5. D'après un entretien avec le président de l'Association des Industriels de Merter (MESSIAD). Il intègre dans cette catégorie les individus qui viennent prendre commande d'au plus 10 contenairs de marchandises (de 20 à $40 \mathrm{~m}^{3}$ chacun).

6. Terme employé à la grande municipalité d'Istanbul lors d'un exposé sur les grands projets urbains (Mai 2000). Les caractères aléatoire et temporaire de la présence de ces populations dans un espace urbain sont un véritable casse-tête pour les aménageurs confrontés aux questions de nomadisme et de sédentarité.

7. Séjours effectués en Février-Mars 2001, Mai-Juin 2001 et Septembre-Octobre 2001.

8. Selon la terminologie employée par A. Tarrius.

9. Le terme « Natacha » est couramment employé pour les désigner ; il signifie « prostituée » en turc.

10. « Argent » en turc.

11. Les policiers syriens tolèrent cette activité désignée comme « illégale ».

12. Le flux de Russes et de Roumains qui constituaient la clientèle la plus importante dans le cadre du commerce de la valise a brutalement chuté en 1998 : cette baisse serait attribuée à la crise économique russe et aux taxes d'importation mais aussi à la concurrence de places commerciales comme la Chine et Dubaï.

13. Dünya Gazetesi, « Bavul ticaretinde ikinci sans » (la deuxième chance du commerce de la valise), 9 mai 2001. 


\section{RÉSUMÉS}

L'article interroge tout d'abord les raisons et les formes d'insertion d'une présence maghrébine dans la métropole stambouliote dans le contexte de fermeture de l'espace Schengen, de dislocation du bloc soviétique et du développement d'une économie de marché en Turquie. L'importance de l'économie informelle dans les pays du Maghreb comme l'ampleur du « commerce à la valise » à Istanbul sont autant d'éléments introductifs à la mise en place d'une chaîne migratoire entre ces deux zones géographiques.

L'article se penche ensuite sur le rôle des résidents Algériens et Tunisiens en présence à Istanbul depuis plusieurs années et leur association avec une population migrante originaire du Sud-est anatolien enrichie dans le commerce international du textile et implantée dans la zone commerciale historique d'Istanbul. Il interroge enfin les répercussions de ces mobilités sur les villes de départ (marchés urbains à Tripoli et Alger en relation avec la centralité marchande d'Istanbul) en situant le trabendiste comme un acteur économique et comme le promoteur de nouveaux agencements territoriaux.

Between the Maghreb and Turkey: Territorial mobility and recomposition. In the first place, the article analyses the reasons and the forms of insertion of North Africans in the metropolis of Istanbul in the context of the Schengen space closure, the collapse of the former Soviet bloc and the building up of a market economy in Turkey. The importance of the informal trade in these countries as the extent of the "suitcase trade » in Istanbul are as many introductory factors to the building up of a migratory network between these two areas.

After the article studies on the role of the Algerian and Tunisian residents in Istanbul since few years and their association with a migrant population coming from the South-East of Anatolia, enriched by the international trade of textile and living in the historical and commercial area of Istanbul. Finally the article raises the question of the repercussions of these mobilities on the departure cities (urban market in Tripoli and Algiers in relation with the commercial centrality of Istanbul) placing the "trabendiste" as an economic actor and as the promoter of new territories.

Movilidades y recomposiciones territoriales. En primer lugar, el artículo analiza las razones y las formas de inserción de la población magrebí en la metrópolis de Estambul en un contexto marcado por el cierre del Espacio de Schengen, el desmembramiento del bloque soviético y el desarrollo de la economía de mercado en Turquía. La importancia de la economía informal en los países del Magreb así como del « negocio de la maleta » en Estambul son factores que presagian la construcción de una red migratoria entre ambas zonas geográficas.

El artículo aborda, a continuación, el papel que juegan los residentes argelinos y tunecinos instalados en Estambul desde hace varios años, así como los lazos que éstos mantienen con la población inmigrante originaria del Sureste de Anatolia. Enriquecida con el comercio internacional de tejidos, esta población se ha implantado en la zona comercial histórica de Estambul.

En último lugar, el artículo trata las repercusiones que estas movilidades pueden acarrear en las ciudades de partida (mercados urbanos de Trípoli y de Argel en relación con la posición comercial central de Estambul). En este sentido, el «trabendiste » constituye un actor económico y es el promotor de nuevos territorios. 
AUTEUR

HÉLÈNE DELOS

Doctorante en géographie, Diasporas, Université de Toulouse-Le Mirail. 